\title{
Declining incidence of osteoporotic hip fracture in Australia
}

1st Author Name: Alice Crisp

Affiliation: National Centre for Monitoring Arthritis and Musculoskeletal Conditions, Australian Institute of Health and Welfare

Address: 26 Thynne St, Fern Hill Park, Bruce, ACT, 2615, Australia

2nd Author Name: Tracy Dixon

Affiliation: National Centre for Monitoring Arthritis and Musculoskeletal Conditions, Australian Institute of Health and Welfare

Address: 26 Thynne St, Fern Hill Park, Bruce, ACT, 2615, Australia

3rd Author Name: Graeme Jones

Affiliation: Menzies Research Institute Tasmania, Hobart

Address: Menzies Research Institute, Private Bag 23, Hobart, TAS, 7001, Australia

4th Author Name: Robert G Cumming

Affiliation: School of Public Health, Anzac Research Institute, University of Sydney

Address: Anzac Research Institute, Concord Hospital (C25), Concord, NSW, 2139, Australia

5th Author Name: Laura L Laslett

Affiliation: Menzies Research Institute Tasmania, Hobart

Address: Menzies Research Institute, Private Bag 23, Hobart, TAS, 7001, Australia

6th Author Name: Kuldeep Bhatia

Affiliation: National Centre for Monitoring Arthritis and Musculoskeletal Conditions, Australian Institute of Health and Welfare

Address: 26 Thynne St, Fern Hill Park, Bruce, ACT, 2615, Australia

7th Author Name: Adrian Webster

Affiliation: National Centre for Monitoring Arthritis and Musculoskeletal Conditions, Australian Institute of Health and Welfare

Address: 26 Thynne St, Fern Hill Park, Bruce, ACT, 2615, Australia

8th Author Name: Peter R Ebeling (Corresponding author)

Affiliation: NorthWest Academic Centre, The University of Melbourne

Address: NorthWest Academic Centre, The University of Melbourne, Sunshine Hospital, Footscray, VIC, 3021, Australia

Email: peterre@ unimelb.edu.au Ph +61383958065 FAX +61383958258

Summary/Mini-abstract: Between 1997-98 and 2006-07 in Australia, the age-standardised incidence rates of hip fractures declined by $20 \%$ and by $13 \%$, in females and males, respectively. Although this may be related to the rollout of public health campaigns and strategies addressing osteoporosis, absolute numbers of hip fractures continued to increase.

The authors have no competing interests. 


\begin{abstract}
Background: Previous reports described an increasing trend in osteoporotic hip fracture incidence in Australia in the 1980s with a stabilisation over the 1990s.

Aim: To describe national trends in the incidence of osteoporotic hip fracture in Australia between 1997-98 and 2006-07.

Methods: Data on low-trauma hip fractures in persons aged 50 years and over were obtained from the National Hospital Morbidity Database. Cases where the patient was transferred in from another hospital were excluded. Age-standardised incidence rates were calculated and a linear test for trend applied.
\end{abstract}

Results: Although the absolute number of hip fracture cases has continued to increase, from 14,769 in $1997-08$ to 16,412 in $2006-07$, these numbers are lower than previous predictions based on population aging. Over the 10-year period, the age-standardised incidence rates in females declined by $20 \%$, from 370 to 295 per 100,000, while the age-standardised incidence rates in males declined by $13 \%$, from 200 to 174 per 100,000 . Both declines were statistically significant. The sex difference in incidence rates narrowed between 1997-98 (females 85\% higher) and 2006-07 (females 70\% higher).

Conclusions: The age-standardised incidence of osteoporotic hip fracture in Australia is falling. This may be related to the uptake of bisphosphonates as well as the rollout of public health campaigns and strategies addressing osteoporosis.

Keywords: osteoporosis, hip fracture, incidence, age-standardised, population health, Australia 


\section{Introduction}

Hip fractures are a major public health problem due to functional impairment, reduced quality of life, pain and disability [1]. For a substantial proportion of sufferers, these functional limitations necessitate a move from independent accommodation to a nursing home, a change that may lead to reduced social interaction, emotional distress, reduced self-confidence and loss of dignity [2]. Activity restriction and anxiety due to the fear of falling are also common [3-5]. Furthermore, the risk of death is raised for several years following a hip fracture [6, 7]. Osteoporosis is the major risk factor for hip fractures. This condition is more common in the older age groups; hence the risk of hip fracture is greater among the elderly and is likely to increase with the aging of the population.

The number and rate of hospital admissions for hip fracture increased during the 1980s in Australia $[8,9]$. Boufous et al. noted that the age-standardised hip fracture rate in New South Wales had stabilised over the period 1990 to 2000 [10]. Recent Victorian data show the age standardised incidence of fall-related hip fracture hospitalisations decreased from $600 / 100,000$ in 1998/1999 to 467/100,000 in 2008/2009 — an estimated overall reduction of $25 \%$ and occurred in both women and men [11]. However, Sanders et al. predicted that, even if the age-specific incidence of hip fracture observed in 1994-1995 remains constant into the future, population ageing would result in the overall number of hip fractures increasing by $36 \%$ between 1996 and 2006, and more than doubling by 2026 [12].

The aims of this study were to:

1. Describe the incidence of minimal trauma hip fracture in Australia over the decade 199798 to $2006-07$;

2. Determine whether the observed trend varies with age and/or sex;

3. Compare the observed number of cases with that which would have been expected had the rates in 1997-98 remained stable over the following 9 years; and

4. Investigate changes in the use of bisphosphonates over the period, as a potential influence on hip fracture incidence. 


\section{Methods}

Data on incident hip fractures were obtained from the National Hospital Morbidity Database for the period June 1997 to June 2007. Virtually all hip fractures require hospital care, and thus hospital separations data can be used to reliably estimate the incidence of hip fracture. This study was restricted to people aged 50 years and over, as minimal trauma hip fractures are uncommon before that age [13].

\section{Data sources and data extraction}

The National Hospital Morbidity Database, maintained at the Australian Institute of Health and Welfare, covers virtually all public and private hospitals in Australia [14]. The information is not person-based; instead it relates to episodes of care in a hospital, also known as 'hospital separations'. To reduce double-counting of multiple episodes of care relating to a single event, separations following a transfer from another hospital were excluded, as proposed by Kreisfeld \& Newson [15].

Cases of minimal trauma hip fracture were identified by selecting separations where the principal diagnosis was fracture of the upper femur (ICD-10-AM codes S720, S721 and S722) and the external cause code indicated a low trauma event (ICD-10-AM codes W00, W01, W03-W08, W18, W19, W22, W50, W51, and W548). For the years 1997-98 and 1998-99 a different coding system was in place, namely ICD-9-AM. The code for fracture of the upper femur under ICD-9-AM (code 820) mapped directly to the ICD-10-AM codes. The majority of external cause codes indicating a low trauma event under ICD-9-AM (codes E8801, E8842-E8846, E885, E8869, E887, E888, E9173, E9174, and E9177-E9179) could be mapped directly to the ICD-10-AM codes. However, code W548 in ICD-10-AM had no equivalent in the older system, and codes 887 and 8801 in ICD-9-AM had no equivalent in the newer system. The difference in the set of external cause codes is expected to contribute a $3 \%$ decrease in the number of fractures from 1999-2000 onwards.

An estimate of the June 2001 Australian population by 5-year age group was used to directly age-standardise the incidence in the comparison with the trend over time for males and females [16]. 
Data on the number of subsidised prescriptions for bisphosphonates dispensed in Australia between 1997 and 2007 were obtained from Medicare Australia via the Pharmaceutical Benefits Scheme online database [17].

\section{Statistical analyses}

Annual (financial year) incidence rates for minimal trauma hip fracture were calculated using the hospital separation data and Australian population estimates [16]. The trends in agespecific rates by sex over the time period were calculated. The trends in the rates for males compared with females were directly age-standardised to the June 2001 Australian population. A simple linear regression was applied to the trends in incidence, and the slope of the line was used to determine whether each trend was statistically significant [18]. The results were extracted and analysed using SAS version 9.1 (SAS Institute Inc., Cary, NC, USA), Hyperion System 9 BI+ Interactive Reporting Studio (Oracle Corporation, Redwood Shores, CA, USA), and Microsoft Excel 2003.

\section{Results}

\section{Incidence rates}

Among Australians aged 50 years and over, the age-standardised hip fracture rate declined significantly between 1997-98 and 2006-07, by 20\% in females (from 370 to 295 per 100,000; $\mathrm{p}<0.001$ ) and by $13 \%$ in males (from 200 to 174 per 100,000; p<0.001) (Figure 1). The rate in females was consistently higher than in males, but this difference decreased between 1997-98 and 2006-07 ( $\mathrm{p}=0.003)$. The rate in females was $85 \%$ higher than in males in 1997-98, but by 2006-07 was only $70 \%$ higher.

The incidence rate was stable in those aged 50-59 years, but decreased significantly among those aged 60-89 years. However, there was a significant increase in incidence rates among males aged 90-94 years (from 1,859 to 2,256 per 100,000; $\mathrm{p}=0.049$ ) and among both males and females aged 95 years and over (from 2,619 to 3,495 per 100,000 in males, $p=0.008$; and from 3,289 to 3,780 per 100,000 in females, $p=0.005$ ) (Figures 2 and 3). Although the latter age group is theoretically open-ended, there was no change in the average age at hip fracture within this group over the period.

The incidence rate in females was higher than in males at all ages except among those aged 95 years and over, where the rates were similar between the sexes (Figure 4). For other age 
groups in 2006-07, the rate in females ranged from $37 \%$ to $115 \%$ higher than in males, with the difference being largest in the 65-69 year old age group and generally decreased thereafter.

\section{Number of cases}

An estimated 16,412 hip fracture cases occurred in 2006-07 (4,443 in males and 11,969 in females). This represented an 11\% increase over 1997-98 ( $<<0.001)$, when there were 14,769 cases (3,631 in males and 11,138 in females). The proportion of cases that occurred in males increased slightly but significantly over the 10 -year period, from $24.6 \%$ to $27.1 \%$ ( $<<0.001$ ). The overall rise was driven by increased numbers of cases in the oldest age groups. The number of cases among those aged 80-89 years increased by around one-third, while the number among those aged 90 years or over more than doubled. Numbers among those aged 50-79 years either remained stable or decreased slightly.

Had the incidence rates observed in 1997-98 remained constant over the following 9 years, an estimated 19,241 cases (a 30\% increase) would have been expected in 2006-07 (Figure 5), approximately 3,000 more hip fractures than were actually observed. These projections were more valid for males than for females.

\section{Discussion}

Our study is this is the first study to produce national age-standardised hip fracture rates using national registry data, as other studies have extrapolated rates determined within specific regions across the country. We demonstrate significant decreases in national age-standardised hip fracture rates for both men and women between 1997-98 and 2006-07. The decline was seen across most age groups, with the exception of those aged 50-59 years (among whom rates were stable) and males aged 90-94 years as well as those aged 95 years and over (among whom the rates increased). We also observed a greater decrease in age-standardised hip fracture incidence among females compared with males.

The observation of overall decreases in fracture rates are consistent with observations from America [20], Canada [21], New Zealand [22], and several European countries [23-25] and observations from Victoria over a similar period [11], which demonstrate both a decrease in the age-standardised incidence of fall-related hip fracture hospitalisations [11], and hip fracture rates [26]. However, our observations contrast sharply with previous Australian 
studies reported an increasing trend in age-standardised hip fracture rates prior to 1990 [8, 9], and a stabilisation between 1990 and 2000 [10, 19].

The greater decrease in age-standardised hip fracture incidence among females compared with males also mirrors overseas trends [20-25]. The generally lower prevalence of osteoporosis in males, coupled with the perception that males are at low risk of the condition [27], may have reduced awareness and so decreased the uptake of strategies to prevent osteoporosis and fractures in males.

Despite the decreasing age-standardised incidence rates, the absolute number of hip fractures has continued to rise due to ageing of the population and an increase in the number of persons at risk. However, the increase observed was much smaller than previously predicted, particularly among females. The projections made by Sanders et al. [12] suggested a 36\% rise, from 15,206 cases in 1996 to 20,754 cases in 2006, among people aged 35 years and over. However, hospital separations data for this age range show an increase of $11 \%$ between 1997-98 and 2006-07, from 14,909 to 16,534 cases (data not shown).

The period of an earlier study from New South Wales (NSW) overlaps with the first three years of our study period. There are two reasons why our data differ. Firstly, we only included hip fractures where the external cause code indicated a minimal trauma event. This accounts for our lower hip fracture rates. Secondly, their data are from NSW hospitals, while our data are from all Australian hospitals. This probably accounts for the small difference in trend over the three years - in their data the male and female rates are steady over the three years, while in our data the male rate is steady, but the female rate decreases.

Several explanations can be offered for our observed decrease in age-standardised hip fracture incidence. Foremost among these are increasing availability and uptake of bisphosphonate medications, and the success of public health campaigns aimed at osteoporosis awareness, and at falls and fracture prevention [10, 20-23]. Other potential influences include the widespread use of hormone replacement therapy in the past, the increase in average body mass index, increased use of calcium and vitamin D supplements, and reduced prevalence of smoking [10, 22].

Almost 3.4 million subsidised prescriptions for bisphosphonates were filled in 2007, for an estimated 300,000 users [28]. This represented a 7\% increase on the previous year, and a 
thirty-fold increase since 1997, when around 108,000 subsidised prescriptions were filled (Figure 6). If one assumes that the hip fracture incidence in bisphosphonate users is $1 \%$ per annum and that bisphosphonates reduce hip fracture incidence by an average of 30\%, [27,28] then one can estimate a reduction in hip fracture numbers of around 1,000 per annum. The lack of a decline in older subjects may reflect less access to or willingness to prescribe or take medication for osteoporosis in this age group. In the largest USA survey the age-adjusted incidence of hip fractures in women increased by 9\% from 1986 to 1995, with a dramatic subsequent decline of 24.5\% between 1996 and 2005 [20]. A similar pattern was observed among men, with a $16.4 \%$ rise over the initial decade studied, followed by a $19.2 \%$ decline in the second 10-year period. The impact of pharmacotherapy with bisphosphonates on fracture incidence rates was calculated to be $40 \%$ in this study.

In contrast to the majority of age groups in our study, the oldest old (males aged 90-94 years and those aged 95 years and over) experienced an increasing rate of hip fracture over the study period. This result suggests that medical or public health initiatives which are successful in younger patients are either less effective or less commonly applied in very elderly patients, or that this group is at even higher risk than younger patients (e.g. due to comorbidity or increased rates of falls). Alternatively, this result may indicate a postponement of the fractures avoided in the younger age groups, fracture of a second hip, or a cohort effect.

The major strength of this study was the use of national data sources that cover the entire Australian population. The National Hospital Morbidity Database covers almost every hospital in the country, and the likelihood of capturing all hip fracture cases is high. The main limitation to this method is the potential for over-counting due to multiple records being generated when a person is transferred from one hospital to another. As recommended by Kreisfeld \& Newson [15], we attempted to account for this by excluding separations with an admission mode of 'transfer from an(other) acute hospital', but it is possible that some secondary separations were still counted as incident cases. Although the effect of this would have been to produce inflated estimates of hip fracture incidence, the amount of overestimation could have varied over time, so the impact on our results is not clear. As noted above, the difference in the set of external cause codes is expected only to contribute a $3 \%$ decrease in the number of fractures from 1999-2000 onwards, far less than the observed decrease. Further, the Pharmaceutical Benefits Scheme online database only contains 
information on subsidised medications. For bisphosphonates, however, information on community or private prescription numbers [29] suggests that only less than $1 \%$ of prescriptions for these drugs would not have been subsidised.

In summary, the age-standardised hip fracture incidence in Australia is declining, but the absolute number of fractures continues to increase due to population ageing. Thus, the Sanders et al. predictions of a tripling in hip fracture numbers by 2036 [12] seems unlikely to eventuate. However, this decline will need to be sustained to compensate for the continued increase in the size of the population at risk of hip fracture due to ageing. Of particular concern is the substantial rise in hip fracture rates among males aged 90-94 years, and those aged 95 years and over. The higher level of care often required by the very elderly may result in a substantial increase in the burden hip fractures impose on health services should this trend continue and as the number of people in this age group increases. Although there remains room for improvement in both sexes, the difference in incidence trends between males and females suggests there is a strong incentive to increase both osteoporosis awareness and the uptake of fracture prevention strategies among older Australian men.

\section{Acknowledgements}

This study was funded by the Australian Government Department of Health and Ageing through its Better Arthritis and Osteoporosis Care initiative. The input of the Advisory Committee of the National Centre for Monitoring Arthritis and Musculoskeletal Conditions is gratefully acknowledged. 


\section{References}

1. Boonen S, Autier P, Barette M, Vanderschueren D, Lips P, Haentjens P (2004) Functional outcome and quality of life following hip fracture in elderly women: a prospective controlled study. Osteoporos Int.15:87-94.

2. Illinois Council on Long Term Care (2008) Understanding the transition to life in a nursing home. Illinois Council on Long Term Care. http://www.nursinghome.org/fam/fam_004.html. [Accessed 29 October 2010].

3. Bertera EM, Bertera RL (2008) Fear of falling and activity avoidance in a national sample of older adults in the United States. Health Soc Work 33:54-62.

4. Salkeld G, Cameron ID, Cumming RG et al (2000) Quality of life related to fear of falling and hip fracture in older women: a time trade off study. BMJ 320:341-6. doi:

10.1136/bmj.320.7231341

5. Zijlstra GAR, van Haastregt JCM, van Eijk JTM et al (2007) Prevalence and correlates of fear of falling, and associated avoidance of activity in the general population of community living older people. Age Ageing 36:304-309. doi: 10.1093/ageing/afm021

6. Bliuc D, Nguyen ND, Milch V et al (2009) Mortality risk associated with low-trauma osteoporotic fracture and subsequent fracture in men and women. JAMA 301:513-521. doi: 10.1001/jama.2009.50

7. Farahmand BY, Michaelsson K, Ahlbom A et al (2005) Survival after hip fracture. Osteoporos Int 16:1583-1590. doi: 10.1007/s00198-005-2024-Z

8. Lau EM (1993) Admission rates for hip fracture in Australia in the last decade. The New South Wales scene in a world perspective. Med J Aust 158:604-606.

9. Lord SR (1993) Hip fractures: changing patterns in hospital bed use in NSW between 1979 and 1990. Aust N Z J Surg 63:352-355. doi: 10.1111/j.1445-2197.1993.tb00401.x

10. Boufous S, Finch CF, Lord SR (2004) Incidence of hip fracture in New South Wales: are our efforts having an effect? Med J Aust 180:623-626.

11. Cassell E, Clapperton A. A decreasing trend in fall-related hip fracture incidence in Victoria, Australia. Osteoporos Int. 2012 Feb 21. [Epub ahead of print]

12. Sanders KM, Nicholson GC, Ugoni AM et al (1999) Health burden of hip and other fractures in Australia beyond 2000. Projections based on the Geelong Osteoporosis Study. Med J Aust 170:467-470.

13. Ross PD (1996) Osteoporosis: frequency, consequences and risk factors. Arch Intern Med 156:1399-1411. 
14. AIHW (Australian Institute of Health and Welfare) (2008) Australian hospital statistics 2006-07. AIHW, Canberra .

15. Kreisfeld R, Newson R (2006) Hip fracture injuries. AIHW, Canberra

16. ABS (Australian Bureau of Statistics) (2008) Australian Historical Population Statistics 2008. ABS, Canberra. http://www.abs.gov.au/ausstats/abs@.nsf/mf/3105.0.65.001 [Accessed 29 October 2010]

17. Medicare Australia (2009) PBS Statistics. Medicare Australia. http://www.medicareaustralia.gov.au/provider/pbs/stats.jsp. [Accessed 29 October 2010] 18. Boyle P, Parkin DM (1991) Statistical methods for registries. In: Jenson OM, Parkin DM, MacLennan R et al (eds). Cancer registration: principles and methods. Oxford University Press, London, pp140-142.

19. Chang KP, Center JR, Nguyen TV et al (2004) Incidence of hip and other osteoporotic fractures in elderly men and women: Dubbo Osteoporosis Epidemiology Study. J Bone Miner Res 19:532-536. doi: 10.1359/JBMR.040109

20. Brauer CA, Coca-Perraillon M, Cutler DM et al (2009) Incidence and mortality of hip fractures in the United States. JAMA 302:1573-1579.

21. Jaglal SB, Weller I, Mamdani M et al (2005) Population trends in BMD testing, treatment, and hip and wrist fracture rates: are the hip fracture projections wrong? J Bone Miner Res 20:898-905. doi: 10.1359/JBMR.041231

22. Fielden J, Purdie G, Horne G et al (2001) Hip fracture incidence in New Zealand, revisited. N Z Med J 114:154-156.

23. Chevalley T, Guilley E, Herrmann FR et al (2007) Incidence of hip fracture over a 10year period (1991-2000): reversal of a secular trend. Bone 40:1284-1289. doi: 10.1016/j.bone.2006.12.063

24. Kannus P, Niemi S, Parkkari J et al (2006) Nationwide decline in incidence of hip fracture. J Bone Miner Res 21:1836-1838. doi: 10.1359/jbmr.060815

25. Lofman O, Berglund K, Larsson L et al (2002) Changes in hip fracture epidemiology: redistribution between ages, genders and fracture types. Osteoporos Int 13:18-25. doi: $10.1007 / \mathrm{s} 198-002-8333-\mathrm{x}$

26. Pasco JA, Brennan SL, Henry MJ et al (2011) Changes in hip fracture rates in southeastern Australia spanning the period 1994-2007. J Bone Miner Res 26:1648-54. 
27. Gill T, Taylor A, Leach G et al (2002) Osteoporosis in South Australia: Prevalence, effects and impact. Department of Human Services, Adelaide.

28. Access Economics (2006) Breaking point: the economic cost of not adhering to bisphosphonate treatment for osteoporosis. Access Economics, Canberra.

29. Department of Health and Ageing (2007) Australian statistics on medicines 2006. Department of Health and Ageing, Canberra. 


\section{Figures:}

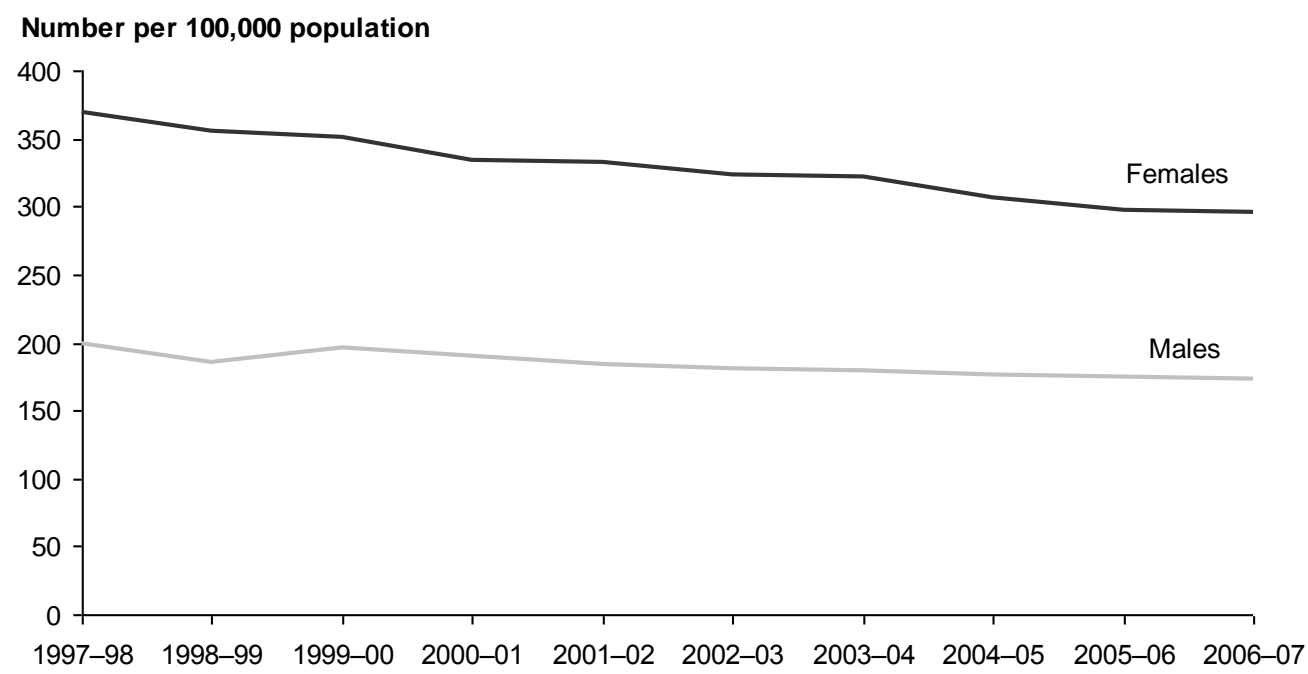

Fig. 1 Trend in osteoporotic hip fracture incidence rates, 1997-98 to 2006-07 (Age-standardised to the June 2001 Australian population) 


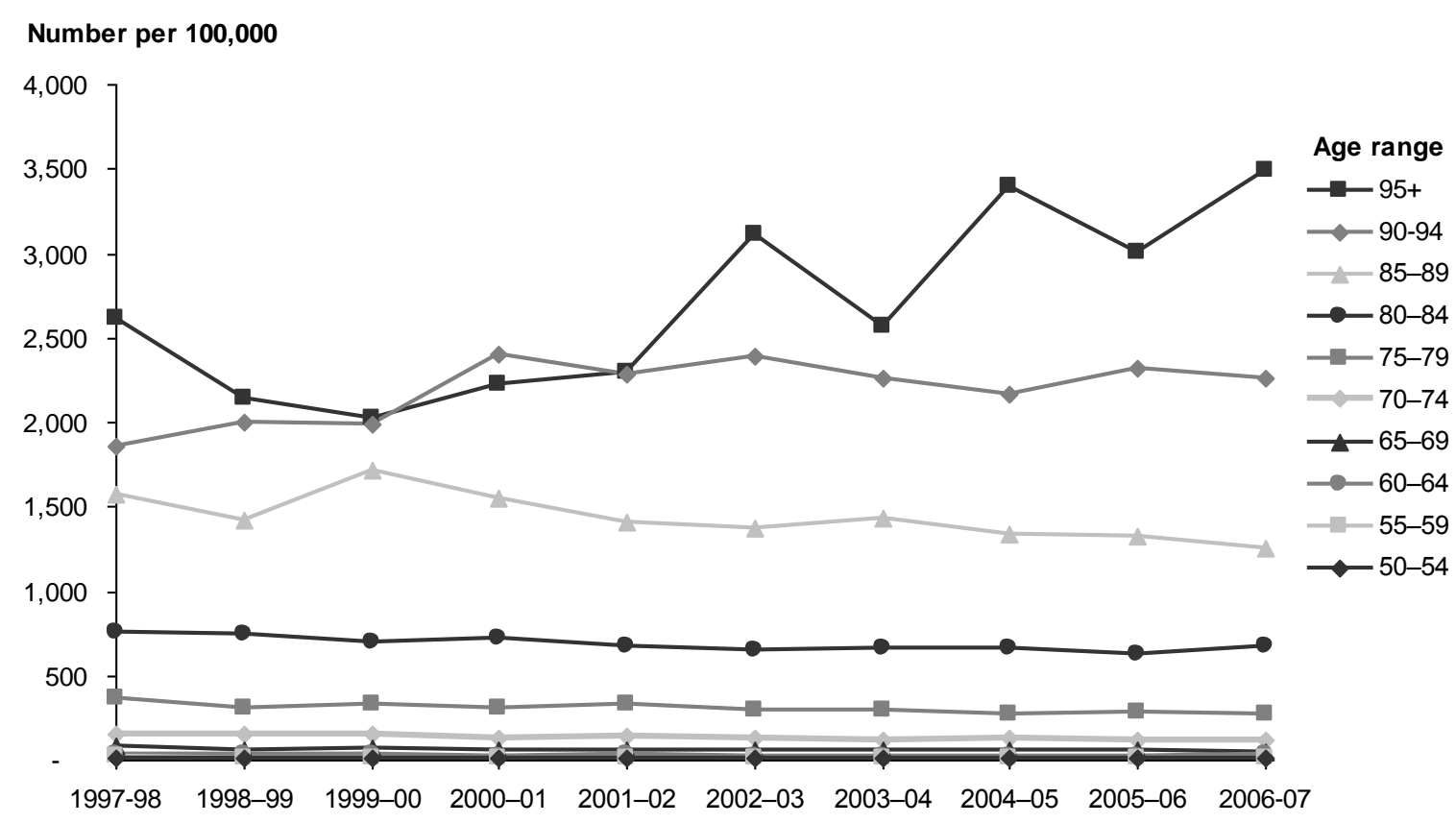

Fig. 2 Trend in osteoporotic hip fracture incidence rates for males by age, 1997-98 to 2006-07 


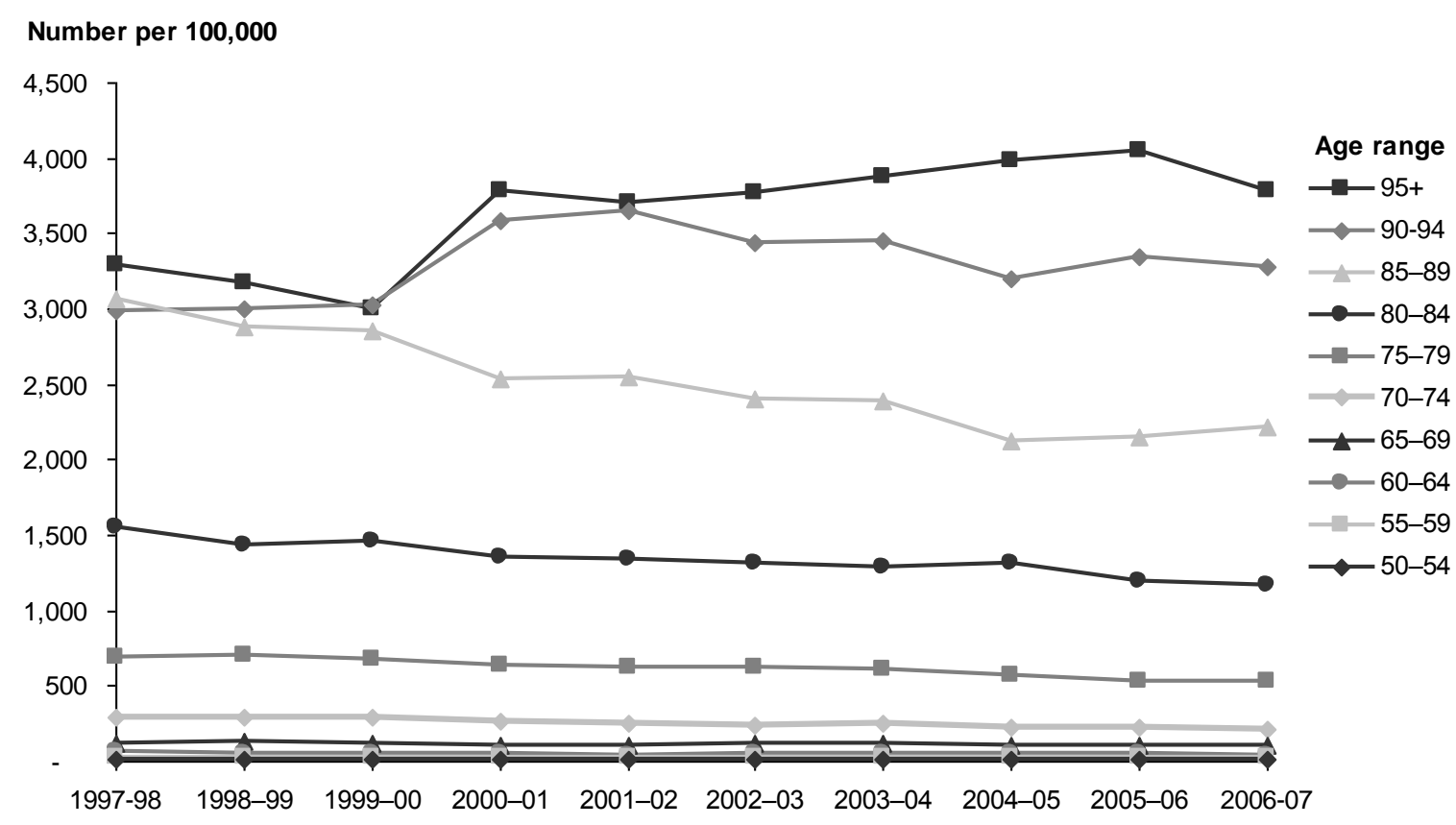

Fig. 3 Trend in osteoporotic hip fracture incidence rates for females by age, 1997-98 to 2006-07 


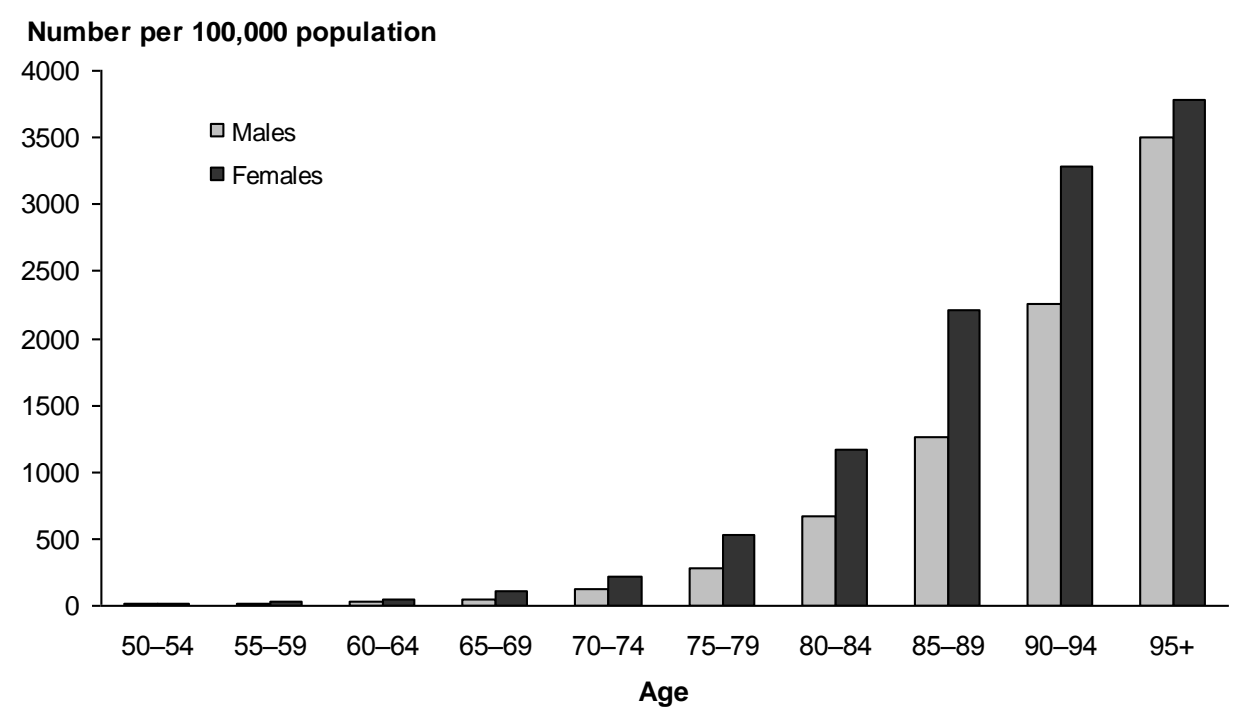

Fig. 4 Osteoporotic hip fracture incidence rates, by age and sex, 2006-07 


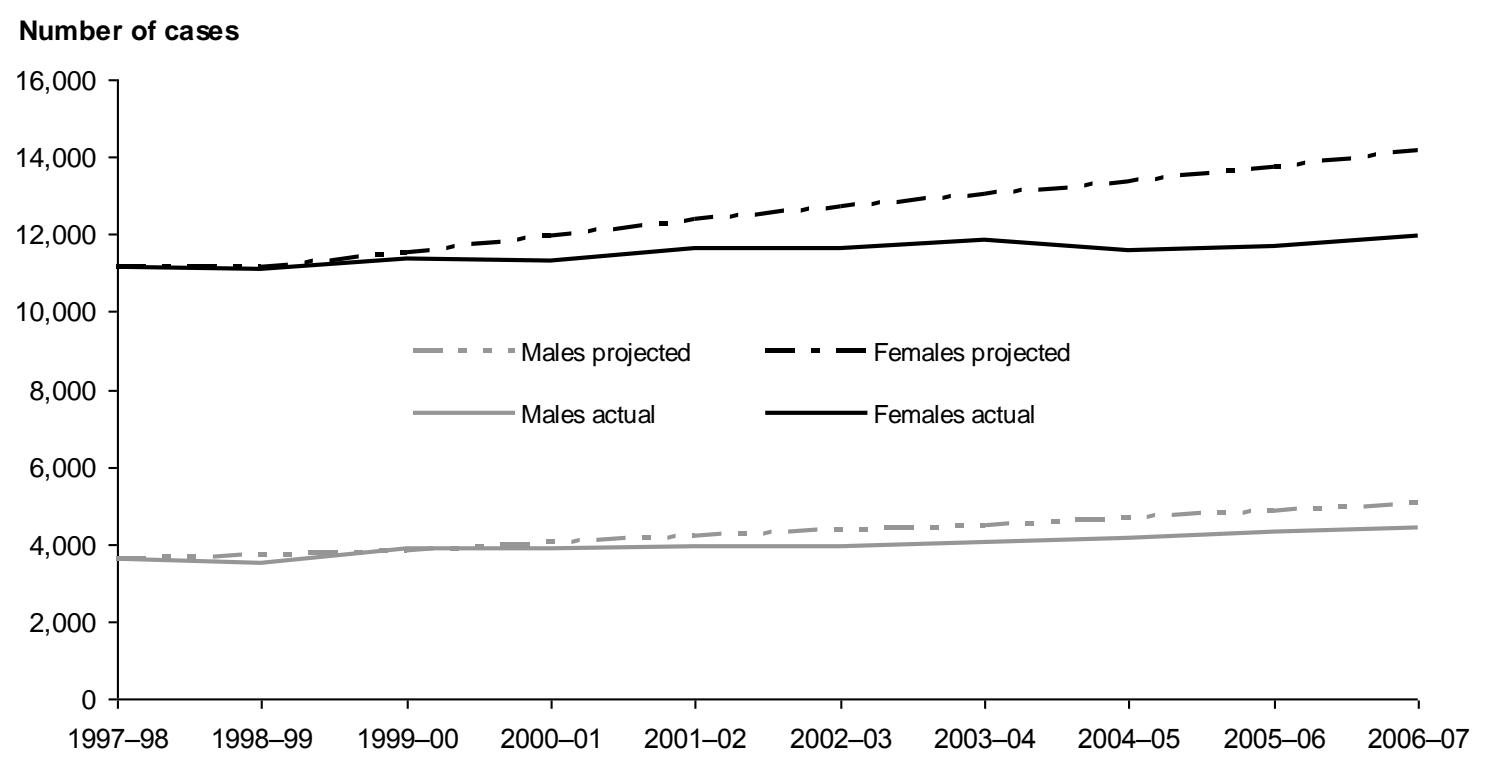

Fig. 5 Actual and projected numbers of hip fractures, 1997-98 to 2006-07 


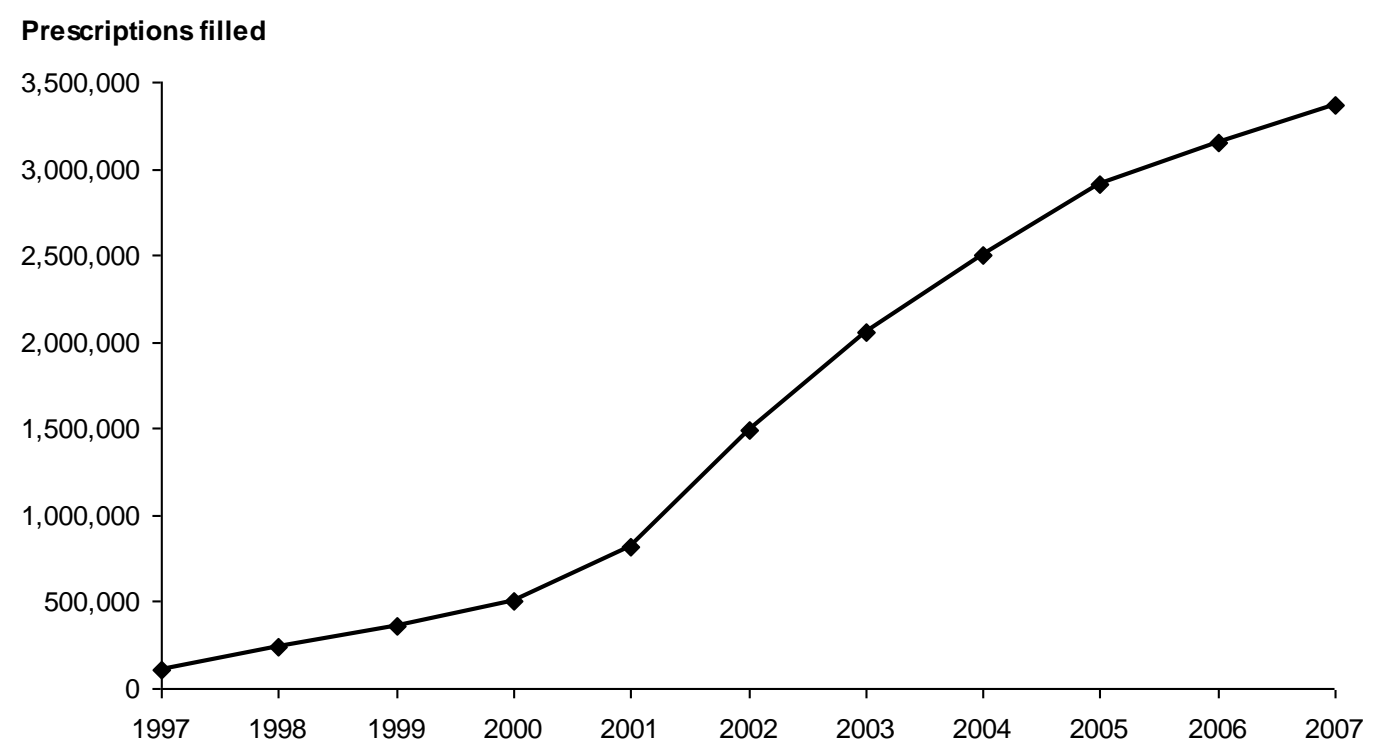

Fig. 6 Subsidised prescriptions for bisphosphonates, 1997 to 2007

(Includes only those prescriptions subsidised through the Australian Pharmaceutical Benefits Scheme) 


\section{Conflict of interest}

No author has any disclosures 